\title{
Faire des bulles, tout un art, toute une science
}

Pierre-Yves Fusier ${ }^{(1)}$, François Graner ${ }^{(2)}$, Frédéric Restagno( ${ }^{(3)}$,

Emmanuelle Rio( ${ }^{(3)}$ (emmanuelle.rio@u-psud.fr), Umar Shoaib ${ }^{(4)}$ et Olivier Vallet ${ }^{(5)}$

(1) Compagnie Slash Bubbles, https://www.slashbubblesparis.com/

(2) Matière et Systèmes Complexes (CNRS et Université de Paris - Diderot)

(3) Laboratoire de Physique des Solides (Université Paris-Saclay et CNRS), 91405 Orsay Cedex

(4) Compagnie Paris Bubbles, www.parisbubbles.com/

(5) Compagnie Les Rémouleurs, www.remouleurs.com

\section{Quoi de commun entre}

un marionnettiste et une doctorante

en physique, un couple de clowns et une physico-chimiste

des matériaux, une vidéaste

et un hydrodynamicien ?

\section{Une passion commune}

pour les films, bulles et mousses

de savon, et une volonté tenace

de construire l'interface art-

science pour que les deux parties

en retirent des idées fructueuses.

\section{Un atelier « Spectacle vivant}

et sciences » a réuni des artistes et des chercheurs en octobre

\section{7 , et à nouveau en novembre}

2019 au Laboratoire de Physique

des Solides, à l'Université

Paris-Saclay.

Accrochez-vous, ça va buller...

Les questions que se posent artistes et scientifiques sont-elles reliées ? Peuventelles faire naitre de nouvelles idées de spectacles ou des questions de physique ? À force d'interagir par petits groupes autour de nos objets, films, bulles et mousses, nous, les auteurs de cet article, avons voulu stimuler les rencontres plus en profondeur. Nous avons invité des chercheurs et artistes du spectacle vivant, presque tous français, et tous travaillant sur les objets savonneux. Chercheurs et artistes étaient en nombre à peu près égal : moins de dix chacun, soit moins de vingt au total.
Un premier atelier, intitulé «Bulles et mousses - À la frontière des sciences et du spectacle vivant ", s'est déroulé à Orsay, dans l'amphithéâtre du Laboratoire de Physique des Solides (LPS), sur deux jours en octobre 2017. Il a commencé par une demi-journée de présentations mutuelles, a enchainé sur de courtes conférences et séances de manipulation, et s'est clos sur une conférence-spectacle pour des collégiens et lycéens. Le tout entrecoupé d'échanges lors des pauses et des repas.

Les questions et réponses croisées ont porté sur la stabilité et la manipulabilité des films, bulles et mousses. Rappelons 
$>>$

qu'un film dit "de savon » est en réalité fait d'eau et d'un tout petit peu de liquide vaisselle : c'est une mince pellicule d'un micromètre d'épaisseur. Une bulle est un film qui est refermé sur lui-même, et qui enferme ainsi de l'air. Une mousse est un assemblage de bulles.

Les discussions et ateliers ont porté sur la taille maximum de ces objets et sur les astuces pour les alimenter. La manière de les rendre visibles et utilisables pour le grand public, tant pour la vulgarisation que pour le spectacle, a fait l'objet d'allersretours avec des participants venus de musées scientifiques. L'hydrodynamique, l'optique, la mécanique, les mathématiques des surfaces minimales et l'acoustique ont été mises à contribution. Les recettes de solutions savonneuses ont été le centre des attentions, avec des discussions sur les méthodes pour les tester, le choix de critères en commun, les échanges des petits secrets de chacun, et une conférence scientifique sur les interactions moléculaires.

Le tout s'est terminé dans l'enthousiasme, et avec une énorme envie de recommencer. Un seul bémol est à signaler : la symétrie entre nous n'a pas été respectée lors de la conférence-spectacle finale. Juxtaposés aux artistes, les scientifiques faisaient pâle figure, semblant parfois briser la magie en expliquant les "trucs » des artistes au lieu de laisser le public apprécier les numéros.

Ce point a été considérablement retravaillé pendant les deux ans qui ont suivi. Établissant des liens étroits par des rencontres régulières associant des doctorantes, trois d'entre nous (P.Y.F., E.R., F.R.) ont construit méthodiquement et testé face au public une conférence-spectacle digne de ce nom où les aspects scientifiques et artistiques se répondent, s'illustrent et se renforcent mutuellement.

Durant ce même intervalle de deux ans, nous avons recensé au moins six nouvelles interactions entre artistes et scientifiques, issues de la rencontre de 2017, portant par exemple sur la durée de vie des films de savon géants, sur des tests systématiques de recettes de solutions savonneuses, sur la construction de mousses suffisamment solides pour tenir debout en soutenant leur propre poids et être sculptables, ou sur des films de savon qu'une musique fait danser.

Une vidéo de quatre minutes nous a permis de rendre compte de l'atelier de 2017, en faisant partager cette première interaction entre scientifiques et artistes.

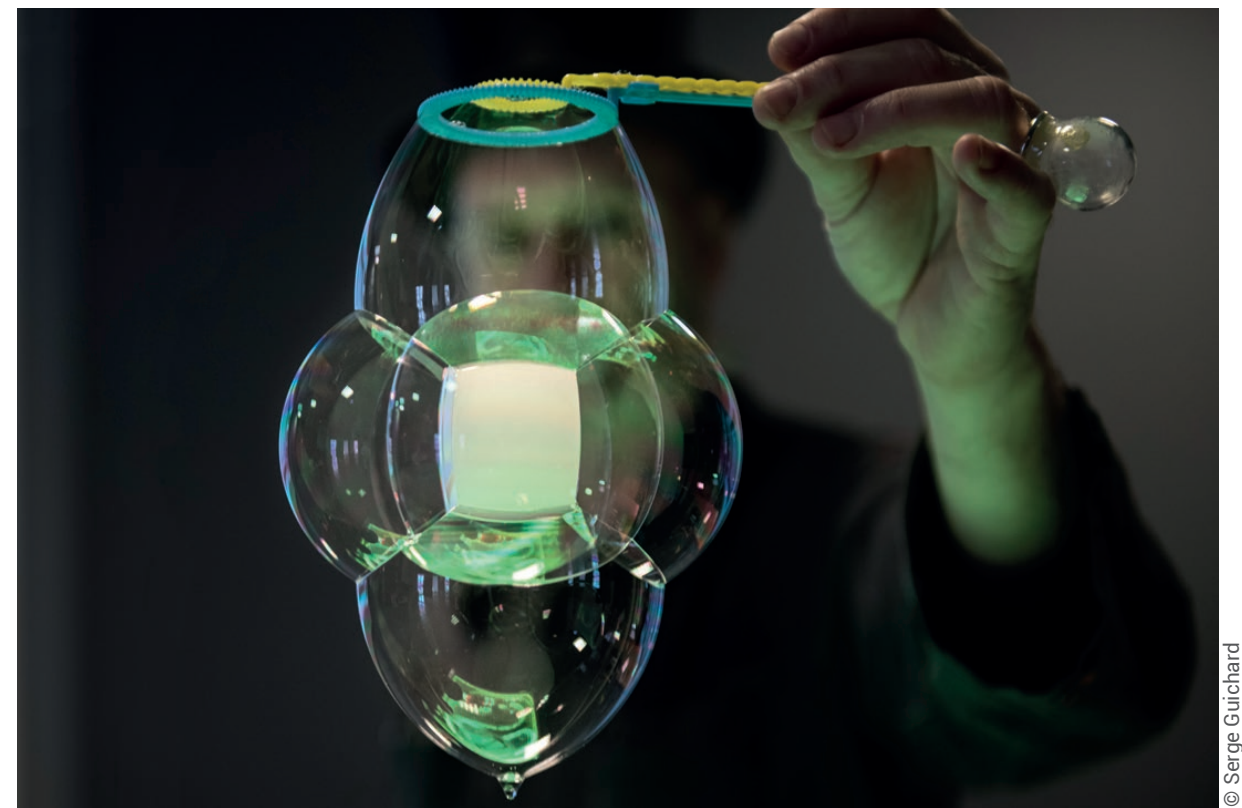

Le cube avec lequel Pierre-Yves Fusier illustre les lois d'assemblage des bulles.

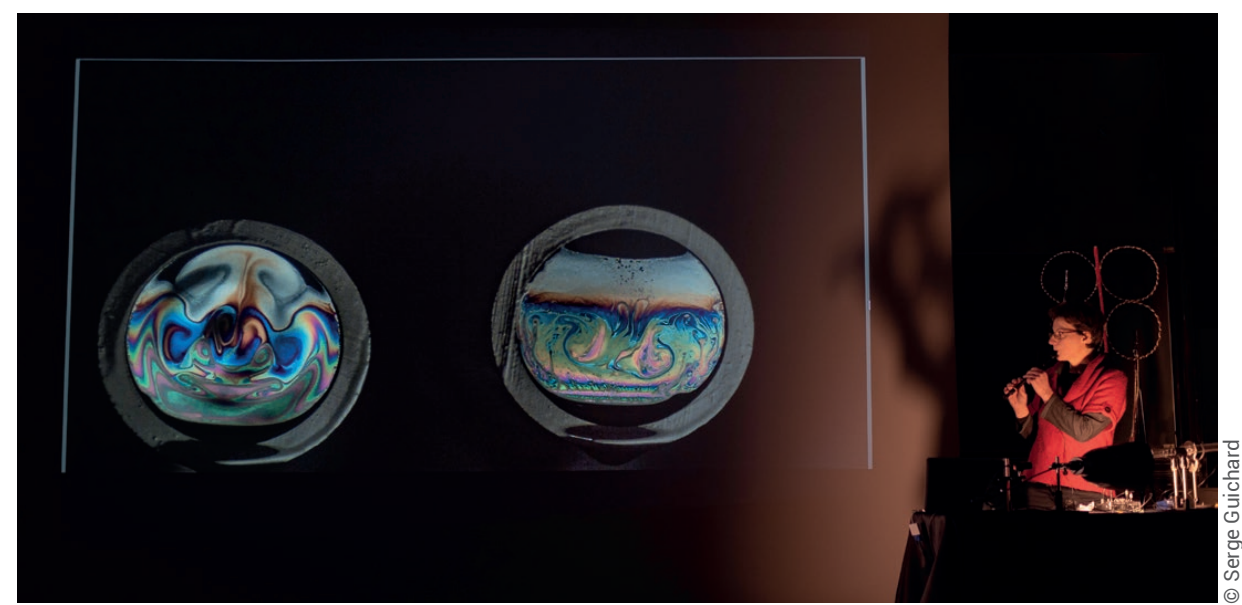

Les films de savon de Florence Elias dansant sur la musique.

Les photos prises pendant l'atelier, ainsi que des photos du travail de P.-Y. Fusier et d'expériences effectuées au LPS, ont été exposées à la Fête de la Science 2019 puis à l'atelier qui a suivi.

Car nous avons bien sûr récidivé, en novembre 2019, pendant trois jours, avec un format légèrement plus grand. Nous avons invité cette fois une trentaine de participants, toujours moitié spectacle vivant et moitié recherche scientifique, européens francophones aux parcours personnels très différents. Les thèmes abordés ont été similaires à ceux de 2017, de façon plus mûre, structurée et variée.

La première demi-journée a été l'occasion de présenter et d'analyser les différentes interactions art-science qui ont été mises en place parmi nous entre 2017 et 2019 .
Trois conférences scientifiques ont couvert la physico-chimie des recettes, la physique de la formation et de l'extension des films, et la stabilité mécanique des mousses. Elles ont permis d'introduire et de préciser un vocabulaire commun, autour des notions de pression, de tension de surface, d'écoulement, d'élasticité d'interface, de compétition avec la gravité, d'évaporation et de viscosité.

Les ateliers ont été plus nombreux, plus longs, coanimés par artistes et scientifiques. Une attention a été accordée à la préparation et aux conditions matérielles, pour permettre de vraies démonstrations et manipulations. Nous nous sommes ainsi retrouvés par petits groupes autour des questions des recettes, de l'alimentation en liquide des films ou des bulles, du spectacle en extérieur, de la médiation scientifique, 
des éclairages, de l'effet du son et du bruit sur les bulles, et des mousses sculptables. Ces deux derniers ateliers se sont d'ailleurs terminés de façon improvisée : ils se sont réunis et ont fusionné pour expérimenter ensemble l'effet de l'acoustique sur des mousses.

Ces trois jours intenses ont été complétés par une discussion sur la dramaturgie, par un hommage à notre regretté collègue physicien Yves Couder, pionnier de l'interface art-science sur les films et bulles, et par une conférence-spectacle qui a clôturé le tout en beauté devant un public de collégiens du voisinage et de collègues du Laboratoire de Physique des Solides.

Les retours que nous ont fait les participants montrent qu'ils se souviendront de cet atelier comme un moment fort de leur carrière. Beaucoup en repartent avec de nouvelles questions, réponses, idées, informations directement utilisables. Des pistes pour le futur sont déjà visibles : une collaboration autour d'un doctorat en cours sur la question de la stabilité des films de savon géants, un spectacle sur les migrants, et un livre de vulgarisation scientifique où de belles photos d'artistes apporteront une réelle illustration aux concepts physiques expliqués. L'avenir nous dira ce qu'il en sortira...

\section{En savoir plus}

Avec le même titre « Faire des bulles, tout un art, toute une science ", ont été réalisés

- le film de l'atelier de 2017, de Max Relid, durée 4'25. Il est disponible sur les sites du Laboratoire de Physique des Solides (LPS) et de Matière et Systèmes Complexes (MSC), et sur www.youtube.com/watch?v=u6Y_594PFcc

- le film de l'atelier de 2019, de Laodice Kolk, durée 3'21. Il est disponible sur les sites du LPS et de MSC, et sur

https://youtu.be/Rf8wo2A3YPg

- I'annonce de l'exposition : une quarantaine de photos de Serge Guichard avec sept panneaux d'explications qui montrent l'atelier de 2017 et ses retombées. Elle a été présentée en 2019 à Orsay (en laboratoire et en ville) et, normalement, devrait l'être très prochainement dans différents sites... À suivre.

Contact : emmanuelle.rio@u-psud.fr

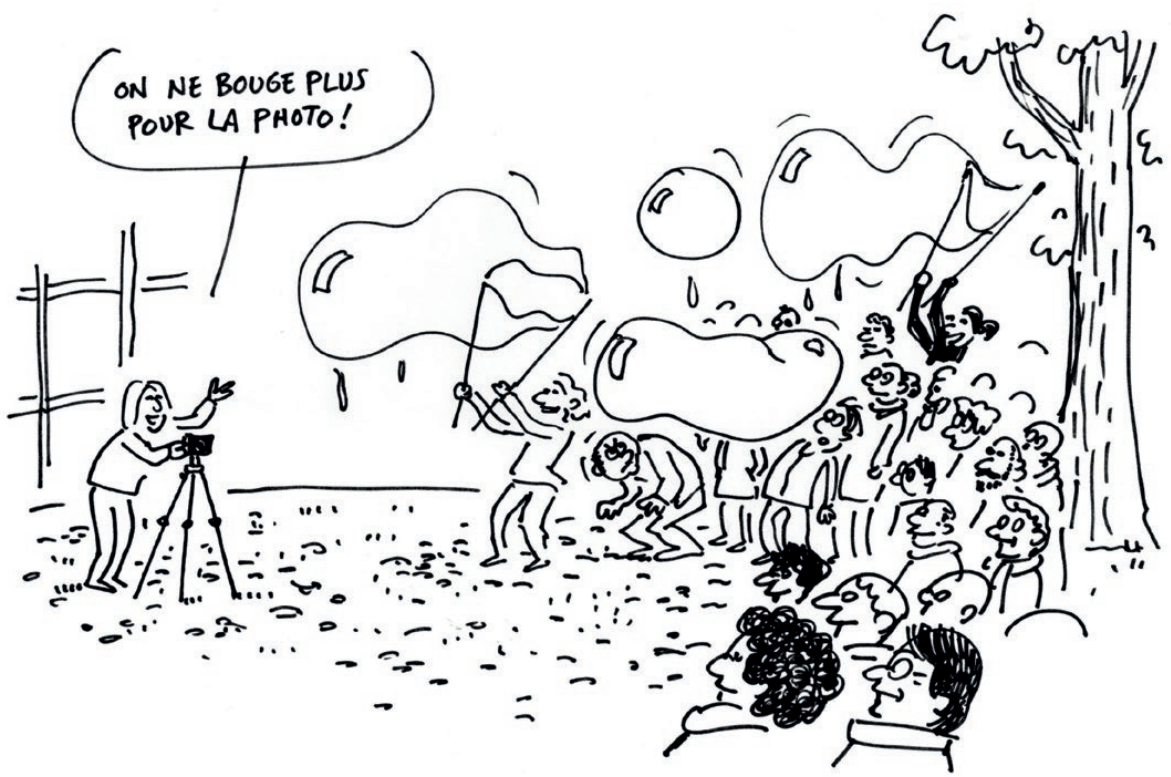

Atelier 2019 : séance de photo de groupe finale sous les bulles géantes, croquée au vol par Jean-Yves Duhoo.

\section{L'organisation en pratique}

P.-Y. Fusier et U. Shoaib, séparément ou en duo, manipulent, assemblent, emboitent ou font éclater des bulles géantes pour des spectacles et des animations dans des contextes variés et inattendus, en intérieur comme en extérieur. E. Rio et F. Restagno travaillent, encadrent des doctorants et font de la vulgarisation dans le domaine de la physico-chimie des interfaces liquides, sur la stabilité des films de savon, des mousses et des émulsions, et sur leurs applications. F. Graner a étudié la structure et les propriétés mécaniques de matériaux cellulaires tels que les mousses de savon; il a utilisé des films et bulles géants en vulgarisation et comme conseiller scientifique sur des spectacles, dont ceux d'O. Vallet, marionnettiste, qui modernise des méthodes anciennes de projections lumineuses analogiques, et qui met en scène des rideaux de savon géants. U. Shoaib a été coorganisateur en 2017. En 2019 il n'a pas pu participer ; nous avons alors été rejoints par O. Vallet.

Lors du colloque de 2019 étaient présents une quinzaine de chercheurs de France et autant d'artistes représentant, pour leur part, non seulement la France entière (région parisienne, Brest, Strasbourg, Toulouse) mais aussi l'Europe, avec des clowns et un bulleur espagnols, un professionnel de la vulgarisation allemand et une fabricante de solutions moussantes du Royaume-Uni. Notre colloque a été accompagné par un dessinateur qui a croqué les participants en direct, deux photographes et une vidéaste.

Nous avons été soutenus par les participants, leur énergie, leur respect mutuel, et l'intensité des collaborations ; par les secrétaires et gestionnaires du LPS pour la préparation et la réalisation ; par le LPS et MSC, I'UFR sciences de l'université ParisSaclay, ainsi que le Labex PALM, qui ont financé le budget à hauteur de $4500 €$ pour le colloque et de $3000 €$ pour l'exposition. Cela a permis de payer les repas de tous, les trajets des artistes, le petit fonctionnement et la vidéo. Les artistes sont bénévoles, hébergés les uns chez les autres ; les missions des scientifiques sont payées par leurs laboratoires. 